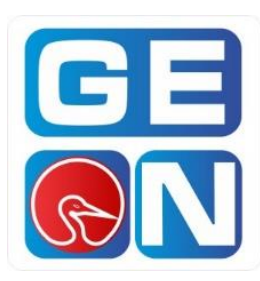

Revista GEON (Gestión, Organizaciones y Negocios.) ISSN: 2346-3910 en línea

revistageon@unillanos.edu.co

Universidad de los Llanos

Colombia

Vázquez Fernández, Enrique; Flores Lujan, Nellyi; Avila, Danae Duana ${ }^{\text {ii }}$.

Planeación de la auditoria administrativa

Revista GEON, Vol. 6, No. 1, 2019

Pág. 37-44

Disponible en: $\underline{\text { https://doi.org/10.22579/23463910.31 }}$

i https://orcid.org/0000-0002-6509-5317

ii $\underline{\text { https://orcid.org/0000-0003-2286-2843 }}$

Esta publicación se encuentra bajo licencia: Creative Commons

ReconocimientoNoComercialSinObraDerivada 4.0 Internacional
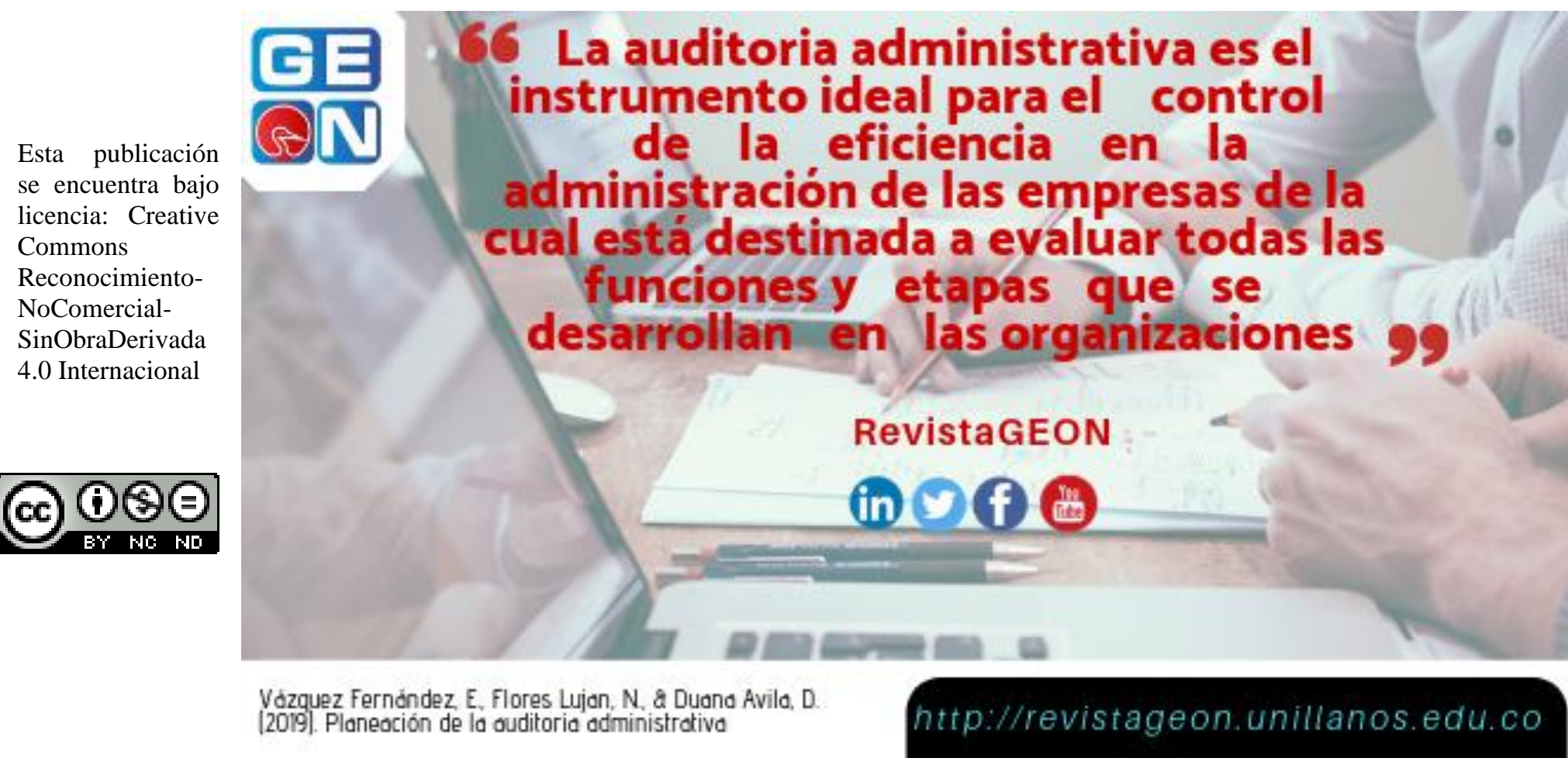


\title{
Planeación de la auditoria administrativa
}

\section{Planning of the administrative audit}

\author{
Enrique Vásquez Fernández \\ envafe@hotmail.com
}

Doctor en Administración, Facultad de Ciencias Económico - Administrativas Universidad Autónoma de Tlaxcala, México

\section{Nelly Flores Luján \\ Angelnes87@hotmail.com}

Mtra. en Administración, Facultad de Ciencias Económico - Administrativas Universidad Autónoma de Tlaxcala, México

\section{Danae Duana Ávila duana@uaeh.edu.mx}

Dr. en economía, Universidad Autónoma de Estado de Hidalgo UAEH, Pe Administración, México

Revista GEON Vol. 6 No 1 enero - junio 2019 ISSN 2346-3910 en linea http://revistageon.unillanos.edu.co/index.php/geon/article/view/31 


\section{Resumen}

Se estudio la fijación del curso de acción que ha de seguirse en el desarrollo de una Auditoria Administrativa, determinando los principios que sirven para orientar y marcar la secuencia de las operaciones necesarias para efectuarla. En primer lugar, vamos a determinar las actividades indispensables de investigación para la planeación de la Auditoria Administrativa, presentándolas en el orden que a nuestro juicio es el más lógico.

En primer lugar, la Investigación preliminar, posteriormente Entrevistas previas y como parte fundamental dos puntos que convergen en la parte medular de este apartado la Definición del área a investigar y Determinación de los detalles a estudiar. investigación

Palabras Clave: Auditoria, investigación, entrevista y área de Abstract
We study the determination of the course of action to be followed in
the development of an Administrative Audit, determining the principles
that serve to guide and mark the sequence of operations necessary to carry
it out. In the first place, we are going to determine the indispensable
research activities for the planning of the Administrative Audit, presenting
them in the order that in our opinion is the most logical.
First, the preliminary investigation, then previous interviews and as a
fundamental part two points that converge in the core of this section the
definition of the area to investigate and determination of the details to
study. area

Palabras Clave: Audit, investigation, interview and research

\section{Code JEL: M1 Business Administration}

\section{Introducción}

Para la auditoria administrativa la planeación e integración de los recursos necesarios especifica en:

1. Determinación del tiempo disponible para la auditoria
2. Selección del personal necesario para efectuarla

3. Documentos auxiliares a solicitar o formular durante la investigación.

Es necesario iniciar el trabajo de obtención de datos con un contacto preliminar una primera idea global. El 
objeto de este primer contacto es percibir rápidamente las estructuras fundamentales y la diferencia principal entre la empresa auditar y otras empresas que se hayan investigado.

El método por seguir será la observación directa de balances, graficas, documentos, estadísticas, etc. Y se ha de iniciar asimilando en lo posible las características de la empresa. Para completar este contacto global es aconsejable visitar cada uno de los departamentos, conocer los productos de la empresa, procesos productivos, los principales mercados, etc.

Es conveniente entrevistar algunas personas directamente relacionadas con la empresa, las cuales deben ser seleccionadas cuidadosamente entre trabajadores, clientes, consumidores, etc. (posiblemente de estas entrevistas surjan algunas ideas o sugerencias acerca de los problemas fundamentales que debemos estudiar durante nuestra auditoria).

En algunos casos esta clase de reuniones dan la posibilidad de planear problemas, descubrir deficiencias en operaciones, funciones o departamentos que están originando alguna dificultad, para que posteriormente en la preparación de cuestionarios se ponga especial cuidado en ellos.

El peligro inmenso y que pocos superen consiste en dejarse llevar por generalizaciones prematuras, en llegar a conclusiones ligeras factibles de comprometer la objetividad de la investigación ulterior.

Si bien es necesario el conocimiento intuitivo del primer contacto, hay que tener en cuenta que solo representa una idea vaga. Es preciso lograr un contacto más consciente, más positivo y fructífero.

El fin de la Auditoría Administrativa es de orientar a la empresa para aumentar su eficiencia, lo cual ha de conducir al mejoramiento social y económico de los integrantes de ella. De ahí la obligación de asociarse con los interesados haciéndoles ver que lo que se persigue es prestar un servicio a la empresa.

No conviene que los integrantes de la empresa auditada tengan la impresión de que los auditores tiene como misión exclusiva la crítica de su trabajo.

Tenemos que definir cuál será la materia objeto de nuestro estudio. Puede ser, desde luego, tola empresa; o bien puede concretarse la revisión a una sola función específica; o bien a un solo departamento, sistema, procedimiento, etc.

Lo ideal es que la revisión abarque toda la empresa con el objeto de formarnos una idea más clara de los problemas desde su origen.

Existen revisiones que comprenden una o más áreas. En estos casos es conveniente examinar, además, aquellas funciones que tengan una relación directa con las áreas auditadas.

Existen diferentes criterios para la determinación de las áreas. Hay casos en los cuales se toma como base para la Auditoria el Proceso Administrativo (Manuel D. Azaola). (2) en otras la revisión se hace en base a las funciones de la empresa (Alfonso Mejía Fernández) (3) o se basa en los niveles jerárquicos, $\mathrm{y}$, por 
último, combinaciones de cualquiera de los enunciados anteriormente (Eugenio Sixto Velazco) (4).

Nuestro personal criterio es que los anteriores solo difieren en la forma mas no en el fondo, dado que cualquiera de ellos puede ser aceptable con la condición de adecuarse a la empresa, a los medios con que cuente el auditor, etc.

Esta Tesis tratamos de enfocarla al Licenciado en Administración, como el profesional natural para desarrollar esta Auditoria consideramos adecuado utilizar como campo de acción las funciones de la empresa, que como ya se mencionó tienen una afinidad con la preparación académica de este profesionista.

A continuación, vamos a presentar un análisis breve de estas funciones.

Es la consecución de los fondos necesarios para estructurar el capital de la empresa mediante la combinación apropiada de recursos provenientes de diferentes fuentes que satisfagan las necesidades del negocio.

1. ¿Se cumplen los objetivos a corto y a largo plazo?

2. ¿Cada cuándo se prevén las necesidades financieras?

3. ¿Cómo se eligen las fuentes de recursos?

4. ¿Son adecuadas las condiciones que se han impuesto a la empresa ala prestar dinero?

5. ¿Las garantías ofrecidas van de acuerdo con el monto de los créditos obtenidos?

6. ¿Es normal la tasa de intereses?
7. ¿Cuál es la base para determinar los plazos para el pago de préstamos?

8. ¿Las utilidades de la empresa son razonables?

9. ¿Cómo está integrado el "superávit"?

10. ¿Existen fuentes internas de recursos?

Es la encaminación, manejo e inversión del capital, en toda la corriente de factores, como instalaciones, maquinaria, equipo, materia prima, salarios, créditos, pagos, etc. Necesarios para el desarrollo de las funciones de la empresa.

1. ¿Se cumplen planes de aplicaciones de recursos?

2. ¿Existen políticas de aplicación de recursos?

3. ¿Son adecuadas las erogaciones por mano de obra, material, gastos de producción, gastos de venta, gastos de administración y otros gastos?

4. ¿Se cumplen oportunamente las obligaciones tributarias?

5. ¿Cómo está integrado el capital de trabajo?

6. ¿Son puntuales las liquidaciones de pasivo?

7. ¿Existen políticas para decretar dividendos?

8. ¿Cada cuándo se formulan y se revisan los presupuestos?

9. ¿Hay un presupuesto maestro y un manual de presupuestos?

\section{Créditos:}


Es el otorgamiento de productos o servicios bajo promesa de posterior pago.

\section{Cobranza:}

Control de cobros e información periódica de los mismos. créditos?

1. ¿Existen políticas para otorgar

2. ¿Son controlados los deudores por estos conceptos?

3. ¿Es oportuna la información?

Etc.

Es la obtención, guarda o entrega de dinero o valores.

1. ¿El control - interno es consistente?

2. ¿Los tramites facilitan las operaciones?

3. ¿Son eficientes las reglas $y$ procedimientos?

Con lo que respecta a el área de personal y reclutamiento consiste en atraer y reunir a un número suficiente de solicitantes a fin de realizar la mejor selección posible del personal adecuado a los puestos.

1. ¿Quién recluta al personal?

2. ¿Se utilizan buenos medios de información para reclutar aspirantes?

3. ¿Se acude a las mejores fuentes de abastecimiento de personal?

Es la función de rechazar a las personas reclutadas, quedándose solo con los elementos que mejor satisfagan las necesidades de los puestos.

1. ¿Se exigen requisitos para los puestos de empleados?
2. ¿Se exigen requisitos específicos para los puestos ejecutivos?

3. ¿Se efectúan exámenes médicos, físicos, psicológicos a los aspirantes?

4. ¿Se comprueban los antecedentes de los solicitantes?

Es la aceptación final del solicitante, formalizándose la relación contractual del mismo, con la empresa.

1. ¿Quién contrata al personal?

2. ¿Cuál es el grado de cumplimiento del reglamento interior de trabajo?

3. ¿Cuál es el grado de cumplimiento de los contratos de trabajo?

En lo que respecta a la inducción se considera el contacto que se establece entre el contratado y la empresa con objeto de adaptarlo lo más rápido y eficazmente posible, al nuevo medio en el cual va a desenvolverse, exponiéndole los objetivos de la empresa, sus políticas, reglas disciplinarias, lugares en los cuales va a trabajar, etc.

1. ¿A los trabajadores de nuevo ingreso se les entrega un manual de bienvenida?

2. ¿Son presentados a sus compañeros los trabajadores de nuevo ingreso?

3. ¿A los trabajadores de nuevo ingreso se les muestran las oficinas, planta y servicios de la empresa?

4. ¿Conocen el reglamento interior de trabajos los trabajadores de nuevo ingreso?

El entrenamiento y desarrollo como ya se mencionó con anterioridad consiste 
en la adecuación de las habilidades del trabajador a las necesidades de la empresa y la optimización de las habilidades potenciales del individuo.

1. ¿Quién entrena o desarrolla al personal?

2. ¿Existen programas de desarrollo para ejecutivos?

3. ¿Existen programas de entrenamiento para empleados?

4. ¿A los nuevos empleados se les entrena?

5. ¿Cómo se elige a las personas a entrenar o desarrollar?

6. ¿Son conocidos los resultados de los programas de entrenamiento $\mathrm{y}$ desarrollo?

7. ¿Se usan medios externos de entrenamiento y desarrollo?

8. ¿Son eficientes los medios internos de entrenamiento y desarrollo?

El análisis y valuación de puestos es la separación y ordenamiento preciso de los elementos que integran un puesto, las tareas que debe abarcar, los requisitos que debe llenar quien lo desempeñe, precisando su misión, obligaciones y su autoridad.

1. ¿Están definidas correctamente los puestos de la empresa?

2. ¿Quién efectúa los análisis?

3. ¿En qué forma se analizan los puestos?

4. ¿Con que periodicidad se analizan los puestos?
Consiste en determinar la importancia relativa de cada puesto, en función de sus obligaciones y responsabilidades, con el fin de asignar a cada uno la retribución y remuneración del personal.

1. ¿Qué elementos se consideran para la evaluación de puestos?

2. ¿Se efectúan comparaciones externas?

3. ¿Intervienen en su elaboración directivos y trabajadores?

4. ¿Cada cuándo se revisan las valuaciones?

\section{Calificación de méritos}

Los méritos consisten en la valoración separada de cada una de las cualidades y realizaciones del trabajador que influyan directamente en la ejecución del trabajo, lo cual permite conocer en el momento necesario a quienes deben darse preferencia en los ascensos, a quienes debe rechazarse fundadamente en los periodos de prueba, que trabajadores pueden escogerse para que ocupen los puestos de confianza, que cualidades pueden ser aprovechadas y desarrolladas en el personal, etc.

1. ¿Cómo se eligen los calificadores?

2. ¿Cuáles son los elementos que se toman en consideración?

3. ¿Qué formas se utilizan para calificar los méritos?

4. ¿Con que periodicidad se revisan las calificaciones?

5. ¿La persona calificada conoce el resultado de la calificación de méritos? 
Las prestaciones son beneficios adicionales que la empresa otorga en favor y provecho de los trabajadores, fuera del salario estipulado y sus complementos directos, con objeto de producirles un mejoramiento económico, social y moral, pudiendo consistir en bienes, instalaciones, facilidades o simplemente en ciertas acciones

1. ¿Otorga la empresa prestaciones y da servicios?

2. ¿El personal conoce los servicios que presta la empresa?

3. ¿Cuáles son las prestaciones $\mathrm{y}$ servicios colectivos?

4. ¿Cuáles son las prestaciones $\mathrm{y}$ servicios personales?

5. ¿Se otorgan prestaciones y se da servicio de carácter familiar?

6. ¿Son utilizados realmente los servicios?

Los salarios por lo tanto se ocupan de las remuneraciones al personal, estudio, implantación y otorgamiento de bonificaciones como estímulo para el mejoramiento e incremento de trabajo, el estudio de modificaciones generales a los salarios, etc.

1. ¿Se estimula el desempeño?

2. ¿Los incentivos se calculan técnicamente?

3. ¿Con qué resultados?

4. ¿Son comprendidos los incentivos por los trabajadores?

5. ¿Los programas de productividad van en relación con estímulos o incentivos?
6. ¿Son adecuados los emolumentos?

7. ¿Se efectúan comparaciones internas?

8. ¿Se efectúan comparaciones externas?

La relación se refiere al logro del mejor espíritu de colaboración posiblemente, mediante la coordinación adecuada entre los fines de la empresa y del personal.

1. ¿Se da un trato adecuado al personal?

2. ¿Se promueve la cordialidad y coordinación entre grupos?

3. ¿Son adecuadas las condiciones de trabajo?

4. ¿Existen sistemas de sugerencias? ¿Se atienden?

5. ¿Existe sistema de quejas? ¿Se atienden?

6. ¿Existe cordialidad en el grupo?

7. ¿Se adaptan rápidamente los nuevos miembros?

8. ¿Es respetada la autoridad?

9. ¿Existe cordialidad entre grupos?

10. ¿Se da reconocimientos al cumplimiento de las obligaciones?

11. ¿Se da cumplimiento con gusto a las obligaciones?

12. ¿Existe cooperación entre grupos?

13. ¿Se cumplen las promesas?

El derecho escalafonario es la necesidad de "clases o grados de salarios" hay que considerar la necesidad de 
coordinar el aumento de salario con el aumento de eficiencia del trabajador o el cambio de puesto. Es la elaboración y aplicación de planes de modificaciones al salario y asignaciones de puestos mediante el análisis adecuado a los casos específicos, considerando los méritos individuales.

Se distingue de la transferencia, ya que esta aplica solamente cambios entre puestos de igual categoría y no mejoras en los puestos y las percepciones establecidas.

1. ¿Conocen los empleados el nivel máximo a que pueden aspirar dentro de la empresa?

2. ¿Existen sistemas de ascensos y promociones?

3. ¿Cuánto tiempo tienen que esperar para el ascenso?

\section{Conclusiones}

Como se ha mencionado la auditoria administrativa es el instrumento ideal para el control de la eficiencia en la administración de las empresas de la cual está destinada a valuar todas las funciones y etapas que se desarrollan en las organizaciones se considera que la revisión tiene que llevarse a cabo por un grupo de especialistas. Que serían las personas más capacitadas para planear dirigir y coordinar todas y cada una de las actividades que serán aplicadas por los especialistas, una de las metas a seguir por los profesionales en esta área debe de obtener los reconocimientos respecto a la valides de sus opiniones tanto como para fines internos de la empresa como externos y que bajo condiciones de factibilidad y plusvalía se vean reflejados en una función totalmente veraz y objetiva.

\section{Referencias}

Pelayo Ma. Carmen (2000), Las principales teorías administrativas y sus enfoques (en línea) consultado (2012). Monografía. COM

G. Steiner (1983). Planeación estratégica (1ra.Edicion), México, CECSA.

Organización internacional de Trabajo (2012), concepto de microempresas (en línea) consultado (2012). http://deconceptos.com

Consultoría grupo kaizen (2009), seis sigmas (en línea) consultado (2012). http://www.grupokaisen.com.

Gustavo (2008), herramientas para planificación de proyectos (en línea) consultado 2012. http://www.empresores.com/foros/8255herramienta-planificacion-de-proyectos.

Saul Trejo Fuentes (2008), Teoría de la organización (en línea) consultado 2012. http://www.gestiopolis.com/administracionestrategica.

Ivan Dimitrie Moyasevich B. (2005), temas de ingeniería industrial (en línea) consultado 2012. http://perso.wanadoo.es

Mgs. Victor Chininin (2010), planeación estratégica (en línea) consultado 2012. . 